\title{
Erratum: Photon isolation effects at NLO in $\gamma \gamma+$ jet final states in hadronic collisions
}

\author{
T. Gehrmann, ${ }^{a}$ N. Greiner ${ }^{b}$ and G. Heinrich ${ }^{b}$ \\ ${ }^{a}$ Institut für Theoretische Physik, Universität Zürich, \\ Wintherturerstrasse 190, CH-8057 Zürich, Switzerland \\ ${ }^{b}$ Max Planck Institut für Physik, \\ Föhringer Ring 6, D-80805 München, Germany \\ E-mail: thomas.gehrmann@uzh.ch, greiner@mpp.mpg.de, gudrun@mpp.mpg.de
}

ERrATUM TO: JHEP06(2013)058

ABstract: We present the NLO QCD corrections to $p p \rightarrow \gamma \gamma j$ production at hadron colliders. Our calculation includes contributions from the fragmentation of a hadronic jet into a highly energetic photon, and consequently allows the implementation of arbitrary infrared-safe photon isolation definitions. We compare different photon isolation criteria and perform a detailed study of the dependence of the $\gamma \gamma j$ cross section on the photon isolation parameters.

ARXIV EPRINT: 1303.0824

After publication of this paper, diphoton production in association with a jet, including the fragmentation component, was re-calculated in ref. [70]. After communication with the authors of ref. [70] it turned out that the original version of our code did not call the correct photon fragmentation functions. This affects figure 3, which we replace by the corrected version below, together with a slightly modified discussion of the comparison of figures 3 and 4 . The effect on the figures other than figure 3 is minor. However, below we also show all the figures based on the corrected version of the code.

Comparing figure 3 and figure 4, it can be seen that the qualitative dependence on the hadronic energy threshold parameters $\epsilon_{c}$ (in fixed cone isolation) and $\epsilon$ (in Frixione isolation) is substantially different. In particular, we observe that the cross section remains almost constant over a large range of $\epsilon_{c}$, while for Frixione isolation, the cross section increases mildly over the interval $0.1<\epsilon<1$. This difference in the qualitative behaviour shows that the parameters can not be translated into each other, since the threshold in the fixed cone isolation is rigid, while the threshold in the Frixione isolation is dynamical, and weighted by the distance to the cone axis. 

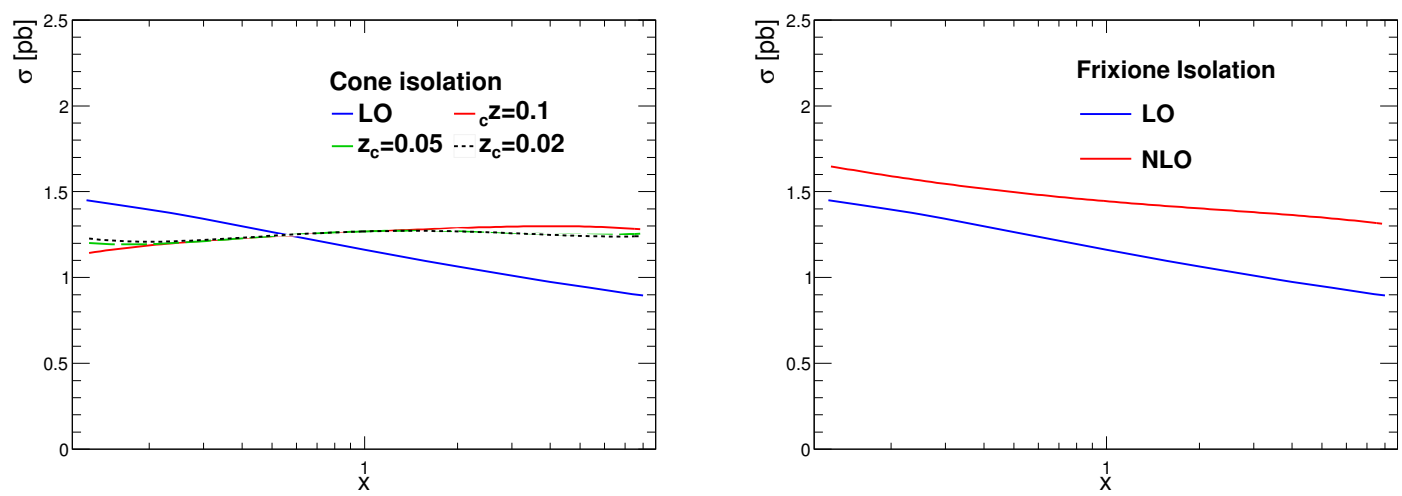

Figure 1. Behaviour of the exclusive $\gamma \gamma+$ jet cross sections with different isolation prescriptions under scale variations, $\mu=x \mu_{0}, 0.5 \leq x \leq 2, \mu_{0}^{2}=\frac{1}{4}\left(m_{\gamma \gamma}^{2}+\sum_{j} p_{T, j}^{2}\right)$.
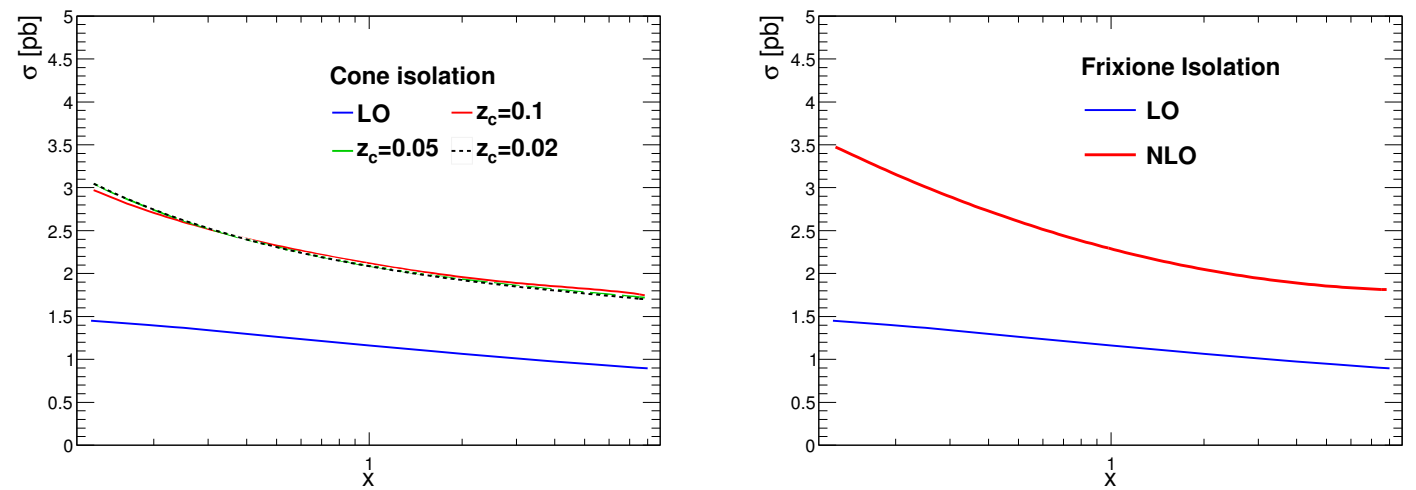

Figure 2. Behaviour of the inclusive $\gamma \gamma+$ jet $+\mathrm{X}$ cross sections with different isolation prescriptions under scale variations, $\mu=x \mu_{0}, 0.5 \leq x \leq 2, \mu_{0}^{2}=\frac{1}{4}\left(m_{\gamma \gamma}^{2}+\sum_{j} p_{T, j}^{2}\right)$.

The pattern of the scale variation band for $\epsilon_{c}$ in the case of exclusive cuts with cone isolation can be understood from the fact that there are several cancellations of scale dependent terms at work. The renormalisation scale dependence acts in opposite direction to the fragmentation scale dependence, i.e. the cross section decreases with increasing $\mu_{r}$, while it increases with increasing $\mu_{F}$. The $\log \left(\mu_{F}\right)$ terms which are contained in the perturbative component of the fragmentation functions largely cancel the $\mu_{F}$ dependence of the direct real radiation part. Only for larger $\epsilon_{c}$ values non-perturbative and beyondleading-logarithmic effects start to become important.

With Frixione isolation, the $\log \left(\mu_{F}\right)$ dependence is absent, therefore the behaviour under scale variations in figure 4 is qualitatively different.

\section{Acknowledgments}

We thank the authors of ref. [70] for pointing us towards this problem. 


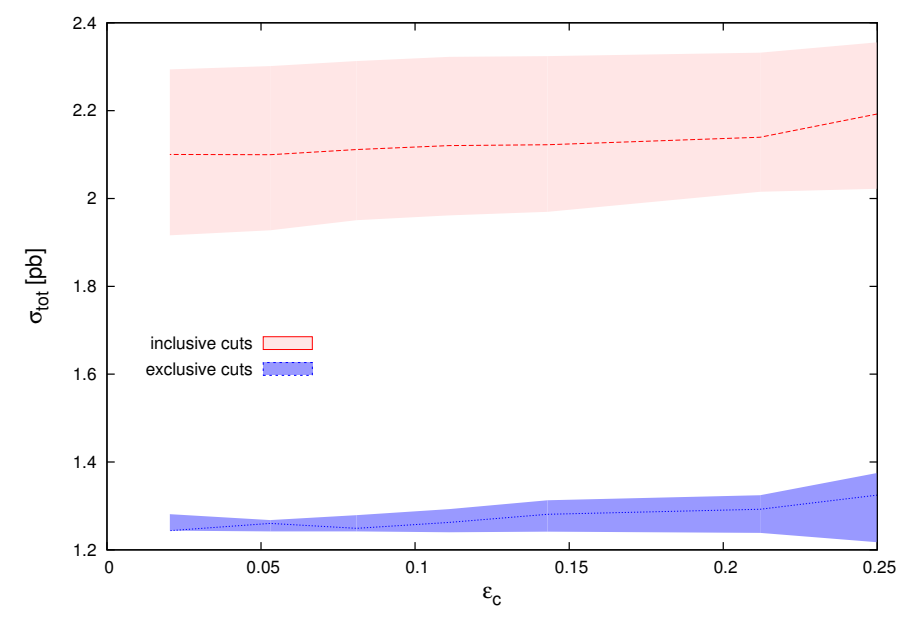

Figure 3. Dependence of the cross sections on the cone isolation parameter $\epsilon_{c}$. The bands correspond to scale variations $0.5 \leq x \leq 2$, with $\mu=x \mu_{0}$.

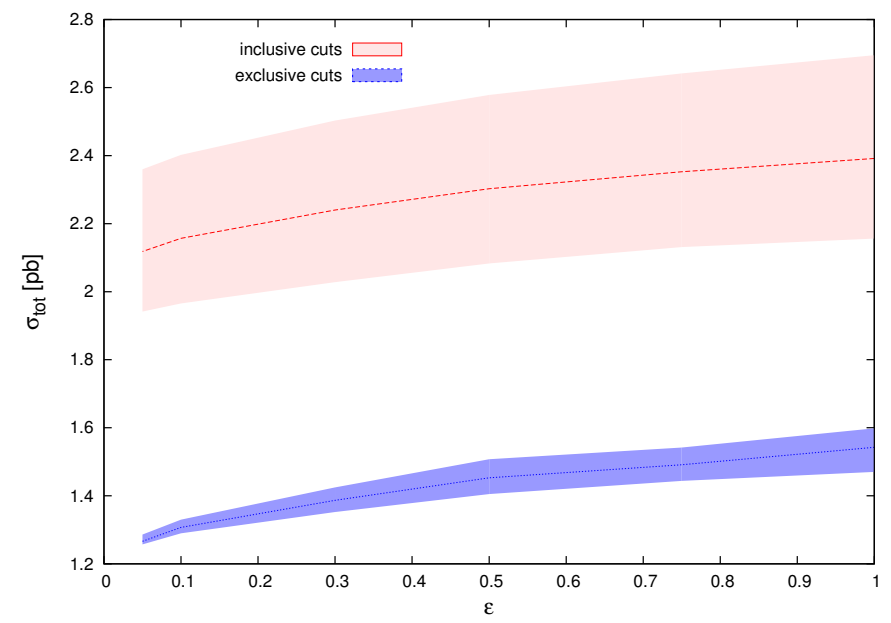

Figure 4. Dependence of the cross sections on the Frixione isolation parameter $\epsilon$.
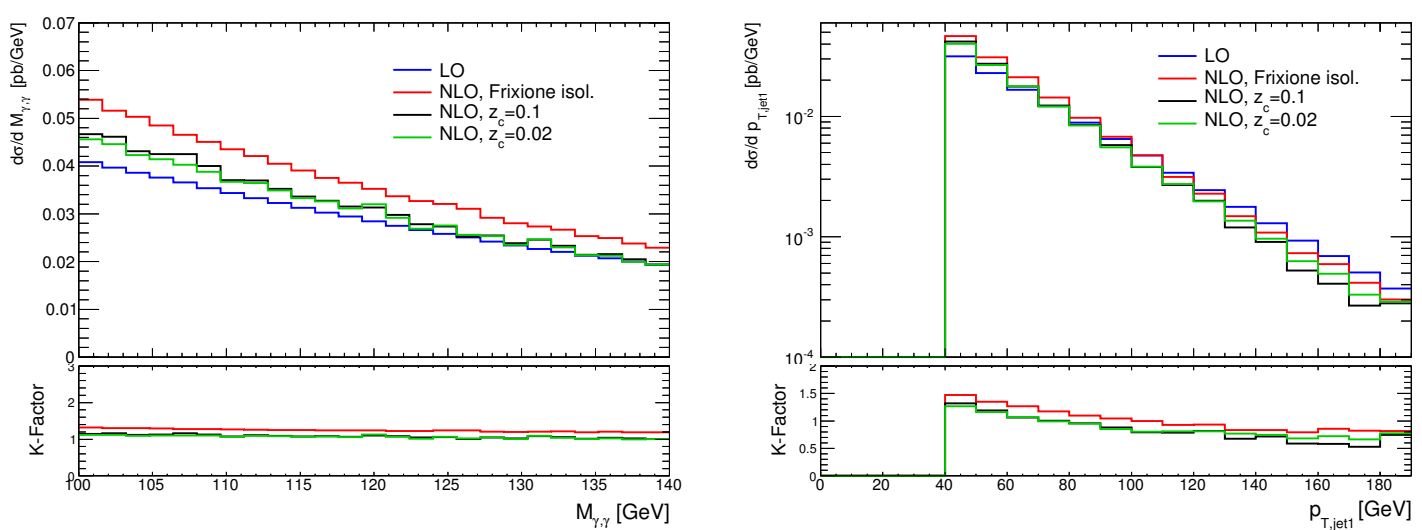

Figure 5. (a) Photon invariant mass distribution, (b) transverse momentum distribution of the leading jet for the diphoton plus one jet exclusive cross section. 

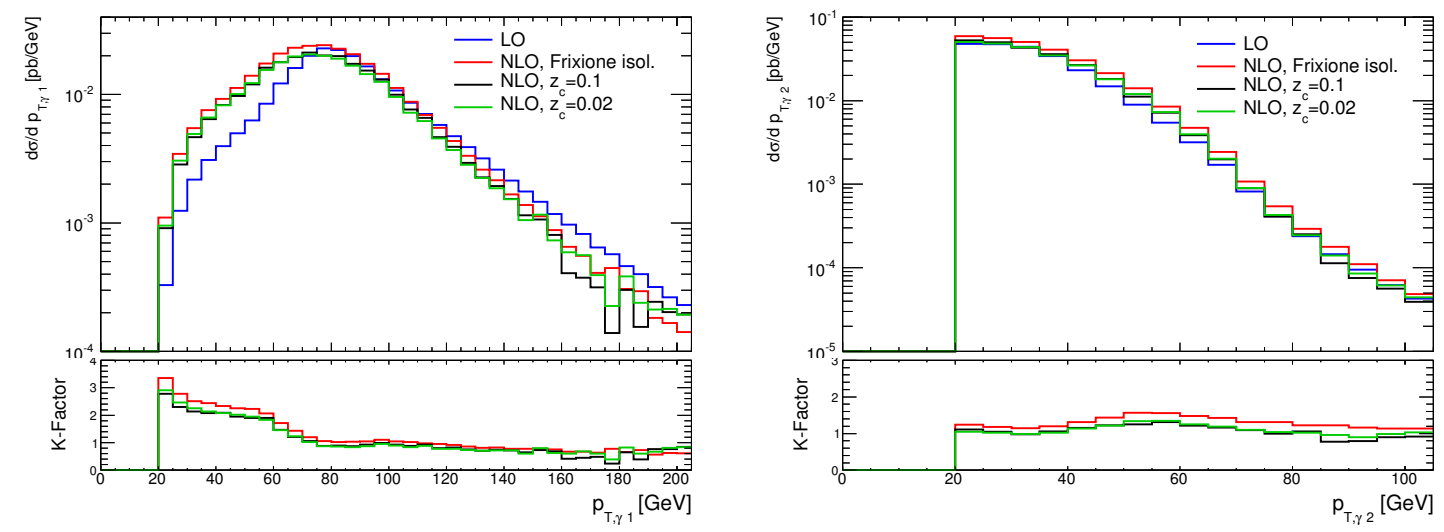

Figure 6. Transverse momentum distributions of the leading (i.e. larger- $p_{T}$ ) and subleading photon for the diphoton plus one jet exclusive cross section.
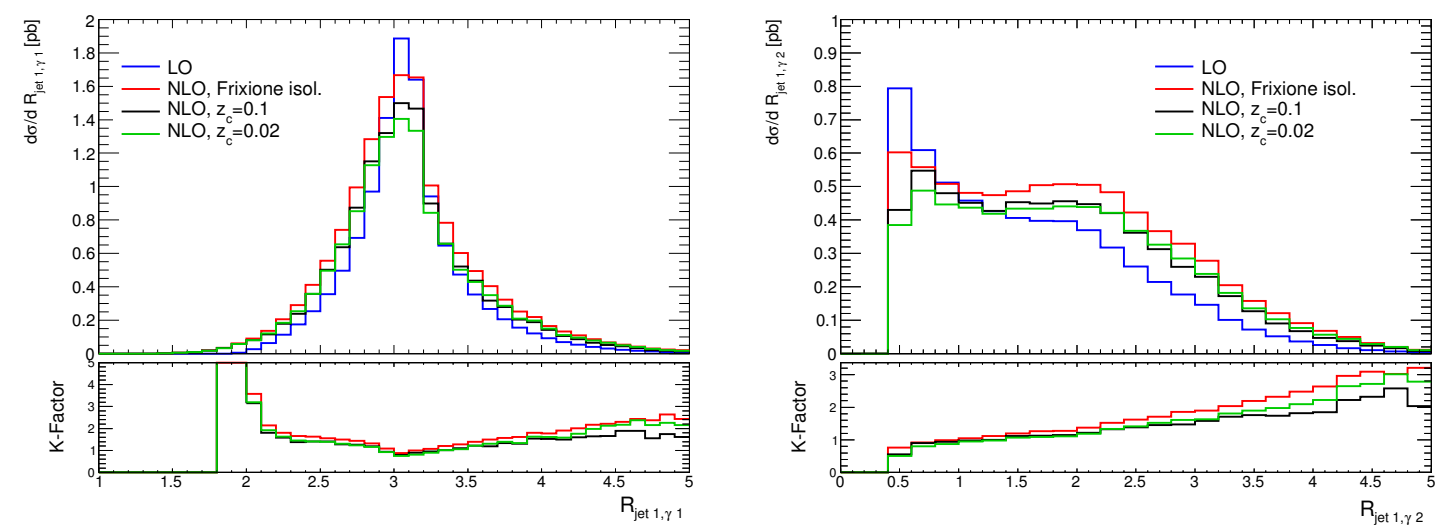

Figure 7. $R$-separation between photon and jet in the $\eta-\phi$ plane for the diphoton plus one jet exclusive cross section. $R_{j e t_{1}, \gamma_{1}}$ denotes the $R$-separation between the jet and the harder photon, while $R_{j e t_{1}, \gamma_{2}}$ is the $R$-separation between the jet and the softer photon.
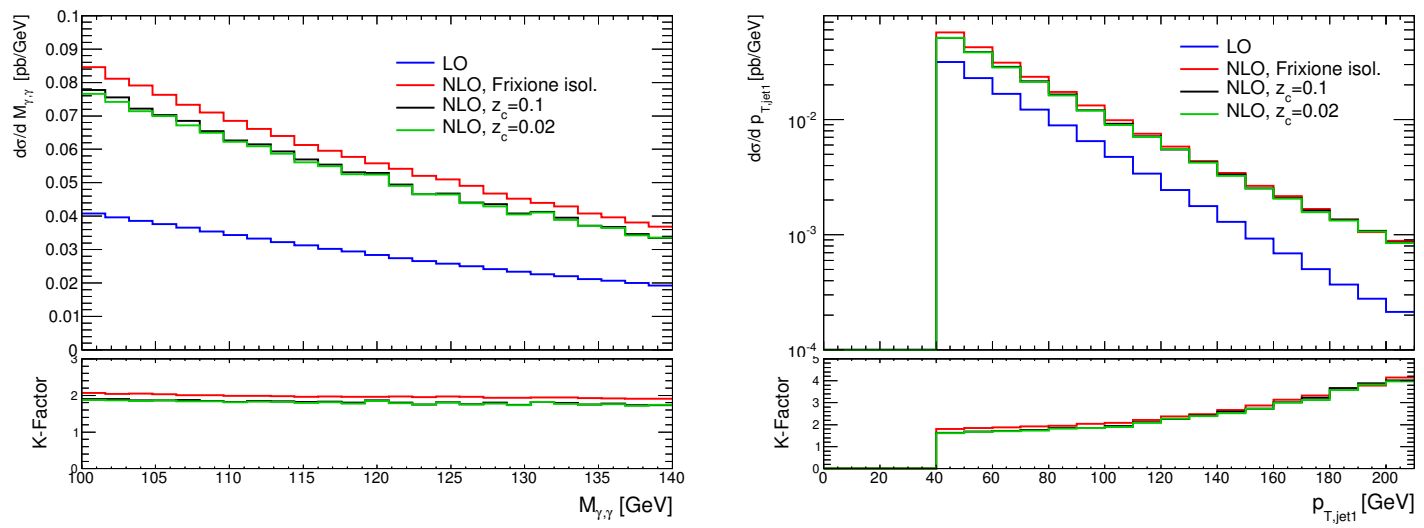

Figure 8. (a) Photon invariant mass distribution, (b) transverse momentum distribution of the leading jet for the diphoton plus one jet inclusive cross section. 

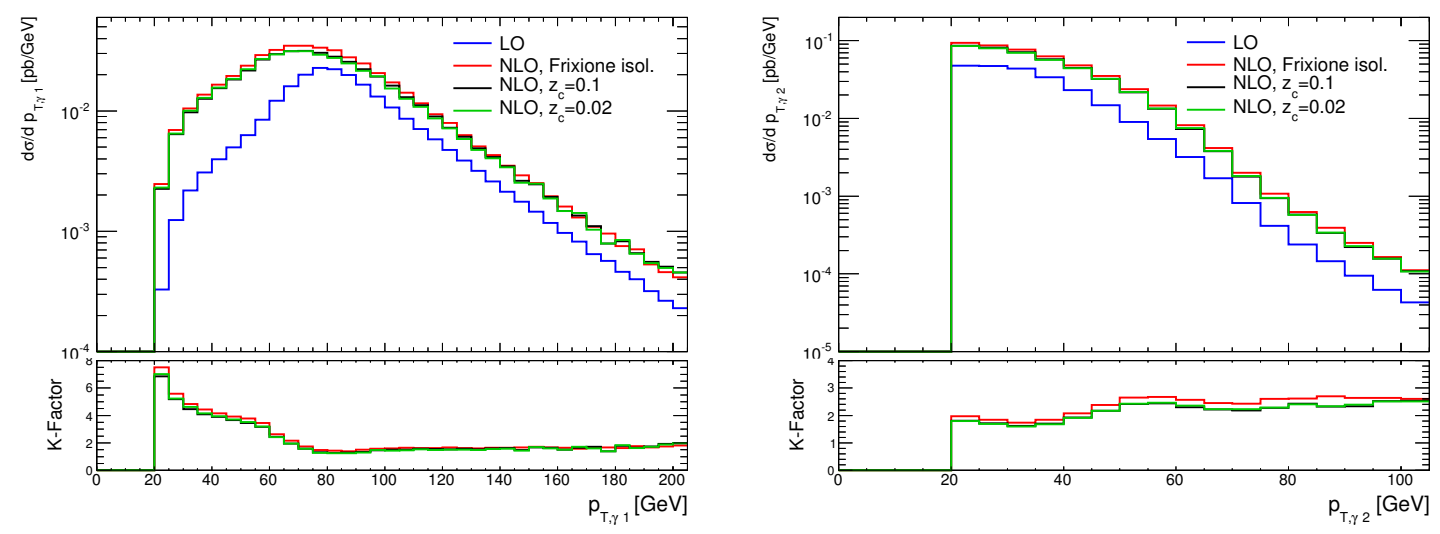

Figure 9. Transverse momentum distributions of the leading (i.e. larger- $p_{T}$ ) and subleading photon for the diphoton plus one jet inclusive cross section.
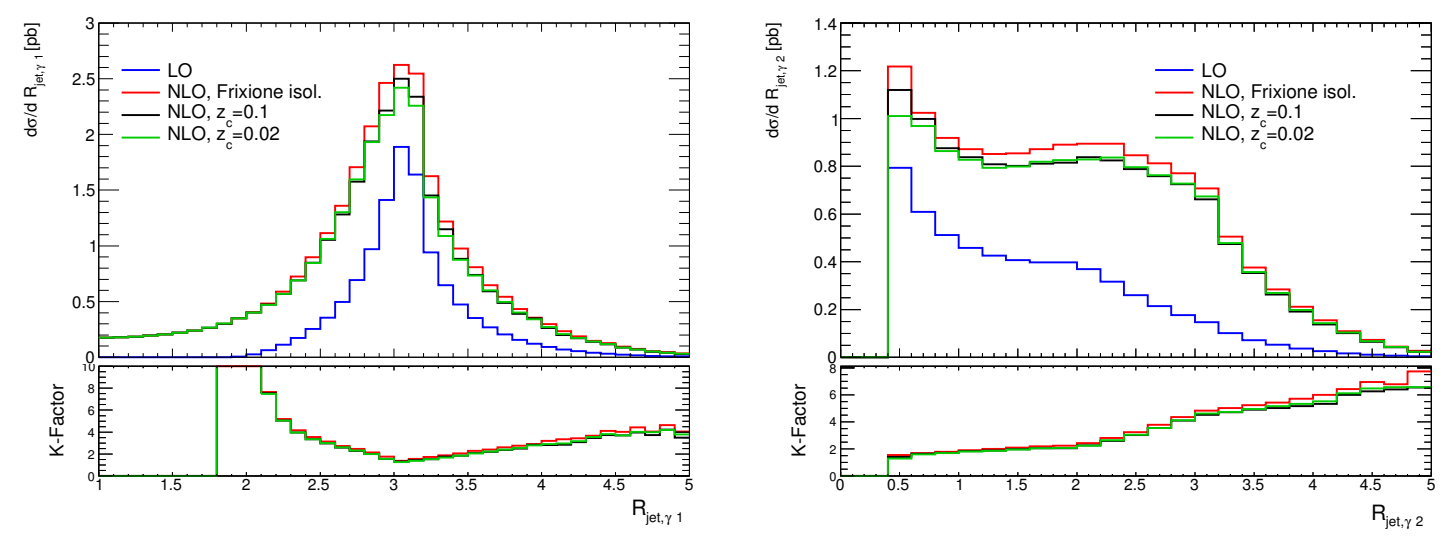

Figure 10. $R$-separation between photon and jet in the $\eta-\phi$ plane for the diphoton plus one jet inclusive cross section. $R_{j e t_{1}, \gamma_{1}}$ denotes the $R$-separation between the jet and the harder photon, while $R_{j e t_{1}, \gamma_{2}}$ is the $R$-separation between the jet and the softer photon.

Open Access. This article is distributed under the terms of the Creative Commons Attribution License (CC-BY 4.0), which permits any use, distribution and reproduction in any medium, provided the original author(s) and source are credited.

\section{References}

[70] J.M. Campbell and C. Williams, Triphoton production at hadron colliders, arXiv:1403.2641 [INSPIRE]. 